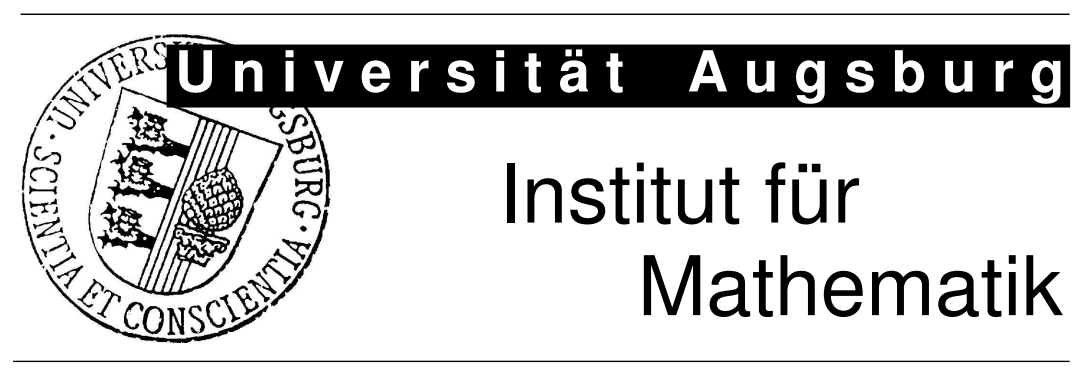

Jost-Hinrich Eschenburg, Peter Quast, Makiko Sumi Tanaka Isometries of Extrinsic Symmetric Spaces 


\section{Impressum:}

\section{Herausgeber:}

Institut für Mathematik

Universität Augsburg

86135 Augsburg

http://www . math. uni-augsburg.de/de/forschung/preprints.html

\section{ViSdP:}

Peter Quast

Institut für Mathematik

Universität Augsburg

86135 Augsburg

Preprint: Sämtliche Rechte verbleiben den Autoren (C) 2015 


\title{
ISOMETRIES OF EXTRINSIC SYMMETRIC SPACES
}

\author{
J.-H. ESCHENBURG, P. QUAST, AND M. S. TANAKA
}

\begin{abstract}
We show that every isometry of an extrinsic symmetric space extends to an isometry of its ambient euclidean space. As a consequence, any isometry of a real form of a hermitian symmetric space extends to a holomorphic isometry of the ambient hermitian symmetric space. Moreover, every fixed point component of an isometry of a symmetric $R$-space is a symmetric $R$-space itself.
\end{abstract}

\section{INTRODUCTION}

Among the compact Riemannian symmetric spaces there is a subclass which always got particular attention: extrinsic symmetric spaces. These are connected submanifolds $M \subset V=\mathbb{R}^{n}$ of some euclidean space $V$ which are invariant under the reflections along any of its affine normal spaces, the extrinsic symmetries. The restriction to $M$ of such a reflection clearly defines a geodesic symmetry of $M$. In particular extrinsic symmetric spaces are Riemannian symmetric spaces w.r.t. the induced metric.

We believe that an extrinsically symmetric embedding $M \hookrightarrow V$ is already generated by the intrinsic structure of $M$. From this philosophy we expect that all isometries of $M$ extend to euclidean motions of the ambient space $V$. This is true:

Theorem 1.1. Let $M \hookrightarrow V$ be an extrinsic symmetric space and $f: M \rightarrow M$ an isometry of $M$ with respect to the induced metric. Then $f$ extends to a euclidean motion $F$ of $V$.

By the very definition of an extrinsic symmetric space, the statement is obvious if $f$ lies in the symmetry group of $M$, this is the closed subgroup of the full isometry group of $M$ which is generated by the geodesic symmetries of $M$. But the full isometry group of $M$ may have connected components where Theorem 1.1 is not obvious at all. This theorem was proved for hermitian extrinsic symmetric spaces in [4] without classification. Although general extrinsic symmetric spaces are just real forms of hermitian ones (see [25]), we could not extend the arguments to the more general case; we had to do a case-by-case study. In fact, isometries of a real form do extend to the hermitian symmetric space (see Theorem 3.1 ), but we can show this only as a consequence of Theorem 1.1.

In Section 2 we give a sharpened version of Theorem 1.1. Sections 3 and 4 discuss consequences. We prove Theorem 1.1 in Section 5 using a general extension principle (cf. Proposition 5.3) and case-by-case arguments. Special attention has to be devoted to the triality automorphism of $\mathrm{SO}_{8} / \pm$, see Section 5.4.

2010 Mathematics Subject Classification. 53C35, 53C40.

Key words and phrases. extrinsic symmetric spaces, isometries. 


\section{EXTRINSIC SYMMETRIC SPACES}

Extrinsic symmetric spaces have been classified by DIRK FERUs [7, 9] (see also [3]): Compact extrinsic symmetric spaces are product embeddings of indecomposable ones, and non-compact extrinsic symmetric spaces are cylinders over compact ones.

Moreover DiRK Ferus [7, 9] has shown that the full compact extrinsic symmetric spaces arise as particular orbits of $s$-representations. Let $M \subset V$ be a full compact extrinsic symmetric space. We may assume that the barycentre of $M$ is the origin of $V$. Since every geodesic symmetry of $M$ extends to an isometry of $V$, the symmetry group of $M$, but also its connected component $K$, the transvection group of $M$, can be considered as a closed subgroup of $O(V)$. By fullness $K$ acts effectively on $V$. As explained in [3], one can extend the Lie bracket on $\mathfrak{k}$ to a Lie bracket on $\mathfrak{g}:=\mathfrak{k} \oplus V$ such that $\mathfrak{g}$ becomes a compact semi-simple Lie algebra and the linear involutive map $\sigma$ of $\mathfrak{g}$ with fixed point set $\mathfrak{k}$ and $(-1)$ eigenspace $V$ is a Lie algebra automorphism. The map $\sigma$ is called Cartan involution. The vector space $V$ is invariant under double Lie brackets and the map $(x, y, z) \mapsto[z,[x, y]]$ for $x, y, z \in V$ defines a Lie triple product on $V$. It turns out that $K$ is the identity component of the group of orthogonal Lie triple automorphims of $V$. If $M$ is indecomposable, then $K$ acts irreducibly on $V$ and $V$ is an irreducible Lie triple. To emphasise the Lie triple structure we will sometimes use the notation $\mathfrak{p}$ instead of $V$. A decomposition $\mathfrak{g}=\mathfrak{k} \oplus \mathfrak{p}$ of a compact Lie algebra into the fixed space $\mathfrak{k}$ and the $(-1)$-eigenspace $\mathfrak{p}$ of an involutive automorphism of $\mathfrak{g}$ is called Cartan decomposition. Up to translation and scaling the extrinsic symmetric space $M \subset V=\mathfrak{p}$ is then the $K$-orbit

$$
M=\operatorname{Ad}_{K} \xi=K \xi \subset \mathfrak{p}=V
$$

of some element $\xi \in \mathfrak{p}$ satisfying

$$
\left(\operatorname{ad}_{\xi}\right)^{3}=-\operatorname{ad}_{\xi}
$$

and vice versa any such orbit is extrinsically symmetric in $\mathfrak{p}$ (see $[6,7,9,3]$ ). From a different point of view these special s-orbits $M$ are known as symmetric $R$-spaces (see [23, $21]$ ), and the extrinsically symmetric embeddings $M \hookrightarrow \mathfrak{p}$ are their standard embeddings $([24,13])$.

Using the notions just established, we can actually sharpen Theorem 1.1 in the case of compact extrinsic symmetric spaces:

Theorem 2.1. Let $f$ be an isometry of a full extrinsically symmetric isotropy orbit $M=$ $K \xi$ contained in $\mathfrak{p}=V$. Then there exists an orthogonal automorphism $\Phi$ of $\mathfrak{g}$ that commutes with the Cartan involution $\sigma$ and preserves $M$ such that $\left.\Phi\right|_{M}=f$.

We assume again that the Lie triple $\mathfrak{p}$ is irreducible. Let $\mathfrak{a}$ be a maximal abelian subspace of $\mathfrak{p}$ that contains $\xi$, and let $\Delta_{+} \subset \mathfrak{a}^{*}$ be a positive root system of $\mathfrak{p}$ corresponding to $\mathfrak{a}$ such that $\alpha(\xi) \geq 0$ for all $\alpha \in \Delta_{+}$. Then $\alpha(\xi) \in\{0,1\}$ for all $\alpha \in \Delta_{+}$. If $\Sigma=\left\{\alpha_{1}, \ldots, \alpha_{r}\right\} \subset \Delta_{+}$ denotes a simple root basis, the value 1 can occur only once since $\delta(\xi)=1$ where $\delta$ is the highest root (which is a sum of all simple roots $\alpha_{j}$ with certain positive integer coefficients $m_{j}$ ). Thus $\xi$ is a dual root of $\Sigma$, more precisely, $\xi=\alpha_{j}^{*}$ for some $j$ with $m_{j}=1$ (see [14, Section 6] and [20, Lemma 2.1]). 


\section{HeRmitian SYMmetric SPACES AND THEIR REAL FORMS}

A case of particular interest happens when $\mathfrak{p}$ is itself a Lie algebra, say $\mathfrak{p}=\mathfrak{g}$, and $\hat{M}=G \xi \subset \mathfrak{g}$ for some $\xi \in \mathfrak{g}$ satisfying Equation (1), where $G$ is the group of inner automorphisms of $\mathfrak{g}$. The tangent space of $\hat{M}$ at $\xi$ is $T_{\xi} \hat{M}=[\mathfrak{g}, \xi]$ and the normal space is

$$
N_{\xi} \hat{M}=[\mathfrak{g}, \xi]^{\perp}=\{\eta \in \mathfrak{g}:[\eta, \xi]=0\}=\operatorname{ker} \operatorname{ad}_{\xi} .
$$

From Equation (1) we see that $\operatorname{ad}_{\xi}$ is a complex structure on $\left(\operatorname{ker} \operatorname{ad}_{\xi}\right)^{\perp}=T_{\xi} \hat{M}$, making $\hat{M}$ a hermitian symmetric space of compact type. This is the standard embedding which assigns to each $x \in \hat{M}$ the complex structure $J_{x}$ on the tangent space $T_{x} \hat{M}$ considered as an infinitesimal isometry fixing $x$ (see $[17, \mathrm{pp} .165 \mathrm{ff}$.$] and [12,9]$ ).

In the previous paper [4] we proved Theorem 1.1 in this particular case: Any isometry $f$ of $\hat{M} \hookrightarrow \mathfrak{g}$ can be extended to an orthogonal Lie triple automorphism $F$ of the ambient Lie algebra $\mathfrak{g}$. More precisely, a holomorphic isometry extends to an orthogonal Lie algebra automorphism, while an anti-holomorphic isometry extends to an orthogonal Lie algebra anti-automorphism of $\mathfrak{g}$.

In the general case, an extrinsic symmetric space $M=K \xi \subset \mathfrak{p}$ is embedded in the hermitian symmetric space $\hat{M}=G \xi \subset \mathfrak{g}$, where $G$ is the group of inner automorphisms of $\mathfrak{g}$. In fact, $M$ is a real form of $\hat{M}$, that is the fixed point set of some anti-holomorphic involutive isometry $f$ of $\hat{M}$. Here we obviously have $F=-\sigma$, where $\sigma$ is the Cartan involution of $\mathfrak{g}$ having $\mathfrak{k}$ as fixed point set. Vice-versa, any real form of a hermitian symmetric space of compact type is actually a symmetric $R$-space. This result is due to MASARU TAKEUCHI [25], see also [22].

Theorem 3.1. Let $M$ be a real form of some hermitian symmetric space $\hat{M}$ of compact type. Then any isometry $f$ of $M$ can be extended to a holomorphic isometry of $\hat{M}$.

Proof. Let $M=K \xi \subset \mathfrak{p}$ be a real form of the hermitian symmetric space $\hat{M}=G \xi$. Let $\mathfrak{g}=\mathfrak{k} \oplus \mathfrak{p}$ be the corresponding Cartan decomposition. Since transvections in $M$ are just restrictions of transvections in $\hat{M}$, we may assume that $f$ fixes $\xi$. By Theorem 2.1, $f$ extends to an orthogonal Lie algebra automorphism $\Phi$ of $\mathfrak{g}$ commuting with the Cartan involution $\sigma$. Recall that $G$ is the group of inner automorphism of the semi-simple Lie algebra $\mathfrak{g}$, which is the identity component of the automorphism group of $\mathfrak{g}$. Thus conjugation by $\Phi$ is an automorphism of $G$. Since $\Phi(\xi)=f(\xi)=\xi$, we have $\Phi(g \xi)=\left(\Phi \circ g \circ \Phi^{-1}\right)(\Phi \xi)=$ $\left(\Phi \circ g \circ \Phi^{-1}\right)(\xi)$ for any $g \in G$. Hence $\Phi$ preserves the adjoint orbit $\hat{M}$, and $\hat{f}:=\left.\Phi\right|_{\hat{M}}$ is an isometry of $\hat{M}$ that extends the isometry $f$ of $M=K \xi$.

Recall that the complex structure of $\hat{M}$ at $\xi$ is given by $J_{\xi}=\left.\operatorname{ad}_{\xi}\right|_{T_{\xi} \hat{M}}$. Let $\hat{f}_{*}$ denote the differential of $\hat{f}$ at $\xi$. Then $\hat{f}_{*}\left(J_{\xi} v\right)=\Phi[\xi, v]=[\Phi \xi, \Phi v]=\left[\xi, \hat{f}_{*} v\right]=J_{\xi}\left(\hat{f}_{*} v\right)$ for all $v \in T_{\xi} \hat{M}$. Using the parallelism of the complex structure $J$, this shows that $\hat{f}$ is holomorphic.

\section{Fixed POINT COMPONENTS IN SYMmetriC $R$-SPACES}

In [5, Theorem 2] we showed that any extrinsically reflective subspace $S \subset M$ of a compact extrinsic symmetric space $M \subset V$, i.e. $S$ is a connected component of $M \cap \operatorname{Fix}(\tau)$ 
for some involutive isometry $\tau$ of $V$ preserving $M$, is itself extrinsically symmetric in $\operatorname{Fix}(\tau)$. The argument can be extended easily to fixed components of any extrinsic isometry (see below). As any intrinsic isometry extends to an extrinsic one by Theorem 1.1, we obtain:

Theorem 4.1. Any positive dimensional fixed point component of an isometry of a symmetric $R$-space is a symmetric $R$-space itself.

Proof. Let $M \subset V$ be a full standardly embedded symmetric $R$-space, and let $f$ be an isometry of $M$. Consider a positive dimensional connected component $Q$ of $\operatorname{Fix}(f)$. By Theorem 2.1, $f$ extends to a linear isometry $F: V \rightarrow V$. Let $V^{+}:=\operatorname{Fix}(F)$ be its $(+1)$ eigenspace. We claim that $Q \subset V^{+}$is extrinsically symmetric and hence a symmetric $R$-space. In fact, let $q \in Q$ and $S_{q}$ the extrinsic symmetry of $M$ at $q$. Observe that $S_{q}$ commutes with $F$ since $F(q)=q$ and hence $S_{q}=S_{F(q)}=F \circ S_{q} \circ F^{-1}$. Then $V^{+}$is $S_{q^{-}}$-invariant and splits into the ( \pm 1$)$-eigenspaces of $S_{q}: V^{+}=V_{+}^{+} \oplus V_{-}^{+}$. Thus $\left.S_{q}\right|_{V^{+}}$is the extrinsic symmetry of $Q \subset V^{+}$at $q$, because $T_{q} Q=V_{-}^{+} \cap T_{q} M$ and $N_{q} Q=V_{+}^{+} \cap N_{q} M$.

\section{Proof of theorems 1.1 AND 2.1}

Let $M \subset V$ be an extrinsic symmetric space and let $f$ be an isometry of $M$. We want to show that $f$ extends to a euclidean motion of $V$. Since by the very definition of an extrinsic symmetric space all geodesic symmetries of $M$ extend to isometries of $V$, and since any two points in $M$ can be interchanged by the geodesic symmetry at the midpoint of a geodesic arc connecting them, we may always assume that $f$ fixes a suitable point of $M$.

\subsection{Reduction to the indecomposable case.}

Recall that by a result of DIRK FERus (see [7, 9] and also [8]) a full extrinsically symmetric embedding $M \hookrightarrow V$ is a product of standardly embedded indecomposable extrinsic symmetric spaces and possibly a Euclidean space $V_{0}$. In particular, $M$ is itself a Riemannian product

$$
M=V_{0} \times M_{1} \times M_{2} \times \cdots \times M_{k},
$$

where the factors $M_{j}, j=1, \ldots, k$ are indecomposable symmetric $R$-spaces. Moreover, as a consequence of Ferus' classification of full indecomposable compact extrinsic symmetric spaces (see $[7,9,3]$ ), one sees the following: The full isometric extrinsically symmetric embedding of a symmetric $R$-space is unique up to extrinsic isometries. Since an isometric permutation of identical factors clearly extends to the ambient space, we only need to prove Theorem 1.1 for indecomposable compact spaces $M \subset V$. For hermitian symmetric spaces of compact type this was done in [4]. Thus we only need to show Theorem 1.1 for indecomposable non-hermitian extrinsic symmetric spaces. These are listed in Table 1 below.

\subsection{Extending isometries.}

The most direct method to verify the claim of Theorem 1.1 is by extending a given isometry $f$ of $M$ directly to the ambient vector space $V$. This applies in particular to the 
TABLE 1. Non-hermitian indecomposable extrinsic symmetric spaces

\begin{tabular}{lllll}
\hline No. & $M$ & $\mathfrak{g}$ & $K$ & Remarks \\
\hline 1 & $U_{n}$ & $\mathfrak{s u}_{2 n}$ & $S\left(U_{n} \times U_{n}\right) / \Delta \mathbb{Z}_{2 n}$ & $n \geq 2$ \\
2a & $S O_{2 n+1}$ & $\mathfrak{s o}_{4 n+2}$ & $S O_{2 n+1} \times S O_{2 n+1}$ & $n \geq 2$ \\
2b & $S O_{2 n}$ & $\mathfrak{s o}_{4 n}$ & $\left(S O_{2 n} \times S O_{2 n}\right) / \pm$ & $n \geq 3$ \\
3 & $S p_{n}$ & $\mathfrak{s p}_{2 n}$ & $\left(S p_{n} \times S p_{n}\right) / \pm$ & $n \geq 2$ \\
4a & $\left(\mathbb{S}^{p-1} \times \mathbb{S}^{q-1}\right) / \pm$ & $\mathfrak{s o}_{p+q}$ & $S O_{p} \times S O_{q}$ & $p+q \geq 3, p$ or $q$ odd \\
4b & $\left(\mathbb{S}^{p-1} \times \mathbb{S}^{q-1}\right) / \pm$ & $\mathfrak{s o}_{p+q}$ & $\left(S O_{p} \times S O_{q}\right) / \pm$ & $p$ and $q$ even \\
5 & $G_{p}\left(\mathbb{H}^{n}\right)$ & $\mathfrak{s u}_{2 n}$ & $S p_{n} / \pm$ & $n \geq 2$ \\
6 & $G_{2}\left(\mathbb{H}^{4}\right) / \mathbb{Z}_{2}$ & $\mathfrak{e}_{6}$ & $S p_{4} / \pm$ & \\
7 & $\mathbb{O}^{2}$ & $\mathfrak{e}_{6}$ & $F_{4}$ & \\
8 & $\left(S U_{8} / S p_{4}\right) / \mathbb{Z}_{2}$ & $\mathfrak{e}_{7}$ & $S U_{8} / \mathbb{Z}_{4}$ & \\
$9 \mathrm{a}$ & $G_{p}\left(\mathbb{R}^{2 n+1}\right)$ & $\mathfrak{s u}_{2 n+1}$ & $S O_{2 n+1}$ & \\
$9 \mathrm{~b}$ & $G_{p}\left(\mathbb{R}^{2 n}\right)$ & $\mathfrak{s u}_{2 n}$ & $S O_{2 n} / \pm$ & $n \geq 2$ \\
10 & $\left(\mathbb{S}^{1} \cdot E_{6}\right) / F_{4}$ & $\mathfrak{e}_{7}$ & $\left(\mathbb{S}^{1} \times E_{6}\right) / \Delta \mathbb{Z}_{3}$ & \\
11 & $U_{2 n} / S p_{n}$ & $\mathfrak{s o}_{4 n}$ & $U_{2 n} / \pm$ & $n \geq 3$ \\
12 & $U_{n} / O_{n}$ & $\mathfrak{s p}_{n}$ & $U_{n} / \pm$ & $n \geq 3$ \\
\hline
\end{tabular}

This list is taken from [2, p. 311], but rearranged according to the order of treatment within this paper. The groups $K$ can be found in [26, p. $324 \mathrm{f}$.$] .$

The notation " $/ \pm$ " means that $x$ and $-x$ are identified.

cases 1, 2 and 3 where $M$ is a matrix Lie group. We need to know the isometry group of a Lie group with a bi-invariant metric.

Lemma 5.1. Let $G$ be a compact connected Lie group with a bi-invariant Riemannian metric and with Lie algebra $\mathfrak{g}=\mathfrak{z} \oplus \mathfrak{g}^{\prime}$ where $\mathfrak{g}^{\prime}=[\mathfrak{g}, \mathfrak{g}]$ is simple and $\mathfrak{z}$ is the center of $\mathfrak{g}$. Further, let $f$ be an isometry of $G$ fixing the identity element e. Then either $f$ or $j \circ f$ is a Lie group automorphism of $G$, where $j: G \rightarrow G, g \mapsto g^{-1}$ is the inversion on $G$.

Proof. Let us first assume $\mathfrak{z}=0$. We follow the arguments given in [16, proof of Theorem 3.3] and [19, Appendix C]. Let $I^{o}(G)$ be the identity component of the isometry group of $G$. We have two injective group homomorphisms $L, R: G \rightarrow I^{o}(G)$ given by $L(g)=$ $L_{g}: x \mapsto g x$ and $R(g)=R_{g^{-1}}: x \mapsto x g^{-1}$, and the group homomorphism $G \times G \rightarrow$ $I^{o}(G):\left(g_{1}, g_{2}\right) \mapsto L\left(g_{1}\right) R\left(g_{2}\right)$ is a covering map. Any automorphism $\phi$ of $I^{o}(G)$ either preserves or interchanges the two subgroups $L(G)$ and $R(G)$. This is because the Lie algebra decomposition $\mathfrak{i}^{o}(G)=\mathfrak{g} \oplus \mathfrak{g}$ is unique and therefore invariant under $\phi_{*}$.

In particular we consider the automorphism $\phi=\phi_{f}$ which is the conjugation by the isometry $f$,

$$
\phi(\alpha)=f \alpha f^{-1}
$$


for all $\alpha \in I^{o}(G)$. If $f$ happens to be the inversion, $f(x)=j(x)=x^{-1}$ for any $x \in G$, then $\phi$ reverses the two factors, that is $\phi(L(g))=R(g)$ and $\phi(R(g))=L(g)$ :

$$
\begin{aligned}
& \phi(L(g)) x=j(g j(x))=\left(g x^{-1}\right)^{-1}=x g^{-1}=R(g) x, \\
& \phi(R(g)) x=j\left(j(x) g^{-1}\right)=\left(x^{-1} g^{-1}\right)^{-1}=g x=L(g) x .
\end{aligned}
$$

If $f$ is arbitrary and $\phi$ interchanges $R(G)$ and $L(G)$, we replace $f$ by $\tilde{f}=j \circ f$, then $\phi_{\tilde{f}}=\phi_{j} \circ \phi_{f}$ preserves these subgroups. Thus we may assume that $\phi$ preserves $L(G)$ and $R(G)$. Then $\phi$ induces automorphisms on these subgroups, $\phi(L(g))=L\left(\phi_{1}(g)\right)$ and $\phi(R(g))=R\left(\phi_{2}(g)\right)$ for two automorphisms $\phi_{1}, \phi_{2}$ of $G$. Using the first of these equations and $f^{-1}(e)=e$, we obtain

$$
\begin{aligned}
& \phi(L(g)) e=L\left(\phi_{1}(g)\right) e=\phi_{1}(g), \\
& \phi(L(g)) e=f(g e)=f(g) \text {. }
\end{aligned}
$$

which proves $f=\phi_{1} \in \operatorname{Aut}(G)$.

In the general case where $\mathfrak{z}$ is arbitrary, we note that the isometry $f$ maps a maximal torus onto a maximal torus. Thus it preserves the center of $G$ which is the intersection of all maximal tori. Hence the differential of $f$ at $e$ preserves the splitting $\mathfrak{g}=\mathfrak{z} \oplus \mathfrak{g}^{\prime}$. The same holds for the inversion $j$. Since any orthogonal map on $\mathfrak{z}$ is trivially an automorphism of $\mathfrak{z}$, we obtain again that $f$ is an automorphism of $G$, maybe after composition with $j$.

5.2.1. Case No. 1: $M=U_{n}$. The first case in Table 1 is $M=U_{n} \subset \mathbb{C}^{n \times n}$. We know by Lemma 5.1: If an isometry $f$ of $U_{n}$ fixes the identity matrix $I$, it is an automorphism $\psi$ of $U_{n}$, maybe after composition with the inversion. Since the inversion $A \mapsto A^{*}$ extends to the ambient space $\mathbb{C}^{n \times n}$, we may assume that $f$ is an automorphism of $U_{n}$. Any automorphism of $G=U_{n}$ leaves invariant the center $\mathbb{S}^{1}=\{z I: z \in \mathbb{C},|z|=1\}$ and the commutator subgroup $G^{\prime}=S U_{n}$. Thus $\left.f\right|_{\mathbb{S}^{1}}$ is an automorphism of $\mathbb{S}^{1}$. There are only two such automorphisms, the identity $z \mapsto z$ and the conjugation $z \mapsto \bar{z}$.

The Dynkin diagram of the semi-simple part $S U_{n}$ (the "string") allows only one nontrivial diagram automorphism, the reflection of the string. Thus any two outer automorphisms differ by an inner one, and one outer automorphism is complex conjugation $A \mapsto \bar{A}$. In fact, its differential is -id on the Cartan algebra of the diagonal matrices in $\mathfrak{s u}_{n}$; this is not a Weyl group element, hence not inner. Thus there are two types of automorphisms of $S U_{n}$, inner ones or inner ones composed with the complex conjugation. Both types extend canonically to $U_{n}$ and to the ambient space $\mathbb{C}^{n \times n}$ : An inner automorphism is the identity on the center $\mathbb{S}^{1}$, and complex conjugation on $S U_{n}$ extends to complex conjugation on $U_{n}$.

It remains to consider the case of "mixed" automorphism which are inner on $S U_{n}$ and complex conjugation on $\mathbb{S}^{1}$ (or the composition of such automorphims with complex conjugation on $U_{n}$ ). If $n \geq 3$, this is not possible since the inner automorphism fixes the intersection $\mathbb{S}^{1} \cap S U_{n}$ but the conjugation on $\mathbb{S}^{1}$ maps $z$ onto $\bar{z}$.

On $U_{2}$ however we also have the automorphism $f: A \mapsto A / \operatorname{det} A$ which is the identity on $S U_{2}$ and the conjugation on $\mathbb{S}^{1}$ since $\operatorname{det} f(A)=(\operatorname{det} A) /(\operatorname{det} A)^{2}=1 / \operatorname{det} A$. Using the inversion formula for $2 \times 2$-matrices $A=\left(\begin{array}{ll}a & b \\ c & d\end{array}\right)$, namely $A^{-1}=A^{v} / \operatorname{det} A$ with $A^{v}=$ 
$\left(\begin{array}{cc}d & -b \\ -c & a\end{array}\right)$, we see that $f$ extends to an orthogonal map $F: X \mapsto\left(X^{v}\right)^{*}$ on the ambient space $\mathbb{C}^{2 \times 2}$, since for $A \in U_{2}$ we have $A^{*}=A^{v} / \operatorname{det} A=f\left(A^{v}\right)$ and therefore $f(A)=\left(A^{v}\right)^{*}$.

5.2.2. Cases No. 2 \&5 3: $M=S O_{n}$ and $M=S p_{n}$. The extrinsic symmetric spaces $S O_{n} \subset$ $\mathbb{R}^{n \times n}$ and $S p_{n} \subset \mathbb{H}^{n \times n}$ are cases 2 and 3 in Table 1. Using Lemma 5.1 again we know that isometries of $M$ fixing the identity matrix are group automorphisms, maybe after composing with the inversion $A \mapsto A^{*}$ which clearly extends to the ambient matrix algebra. Since inner automorphisms also extend it suffices to look for outer automorphisms. The group $S p_{n}$ has Dynkin diagram $C_{n}$ which has no non-trivial diagram automorphisms, thus $S p_{n}$ has no outer automorphisms. The same holds for $S O_{n}$ if $n=2 m+1$ is odd (Dynkin diagram $B_{m}$ ) while $S O_{n}$ for even $n=2 m$ has classes (connected components) of outer automorphisms corresponding to the non-trivial diagram automorphisms of $D_{m}$. For $m \neq 4$ there is just one non-trivial diagram automorphism, and the corresponding class consists of conjugations with any $A \in O_{n}$ with $\operatorname{det} A=-1$, which clearly extend to the ambient matrix space $\mathbb{R}^{n \times n}$.

In the case $m=4$, the group of diagram automorphism is the dihedral group of the equilateral triangle which has two generators, the reflection (which exists for any $m$ ) and the rotation of order 3. An automorphism corresponding to such a rotation is the triality automorphism $\theta$ on $\operatorname{Spin}_{8}$ which does not pass over to $\mathrm{SO}_{8}$, see subsection 5.4. All other elements of this class differ from $\theta$ just by an inner automorphism, hence they are also not defined on $S_{8}$. Thus $n=8$ is no exception: the only outer automorphisms are conjugations with elements of $O_{8}$ whose determinant is -1 , and these extend to the ambient matrix space.

5.2.3. Case No. 4: $M=\left(\mathbb{S}^{p-1} \times \mathbb{S}^{q-1}\right) / \pm \subset \mathbb{R}^{p \times q}$. This is not a group case, but still we can extend each isometry directly to the ambient space. The ambient space is $V=\mathbb{R}^{p \times q}$ with the embedding $\pm(v, w) \mapsto v w^{T}$. The isometry group of $\mathbb{S}^{p-1} \times \mathbb{S}^{q-1}$ is $O(p) \times O(q)$ when $p \neq q$, and if $p=q$, then there is another isometry, the exchange of the two factors. All isometries descend to $M$, and they all extend to orthogonal maps of $V$ : On $V$ we have the representation of $O_{p} \times O_{q}$ by $((A, B), X) \mapsto A X B^{-1}$, and the exchange of the factors (when $p=q$ ) extends to the transposition $X \mapsto X^{T}$ since $v w^{T} \mapsto w v^{T}=\left(v w^{T}\right)^{T}$.

\subsection{Extending automorphisms.}

Let $M \subset \mathfrak{p}$ be a full indecomposable compact extrinsic symmetric space with transvection group $K$. Let $\mathfrak{g}=\mathfrak{k} \oplus \mathfrak{p}$ be the Cartan decomposition of the compact semi-simple Lie algebra $\mathfrak{g}$ explained in Section 2, and let $\sigma$ be the Cartan involution of $\mathfrak{g}$ having $\mathfrak{k}$ as its fixed point set. Recall that up to scaling and translation we have

$$
M=\operatorname{Ad}_{K} \xi=K \xi \subset \mathfrak{p}
$$

with $\xi \in \mathfrak{p} \backslash\{0\}$ satisfying Equation (1).

Since $K$ is the identity component of the isometry group of $M, K$ is invariant under conjugation with any isometry $f$ of $M$. Thus $f$ defines an automorphism

$$
\phi: K \rightarrow K, \quad k \mapsto f k f^{-1} .
$$


Remark 5.2. Recall that we may assume that $f$ fixes $\xi$. Hence $\phi$ leaves

$$
L:=K_{\xi}=\{k \in K: k \xi=\xi\}
$$

invariant.

Instead of extending the given isometry $f$ of $M$ we may as well extend the induced automorphism $\phi$ of $K$. The following theorem shows that this is equivalent.

Proposition 5.3. An isometry $f$ of $M$ fixing $\xi$ extends to an orthogonal map $F$ on $\mathfrak{p}$ if and only if the differential $\phi_{*}$ of the automorphism $\phi$ of $K$ with $\phi(k)=f k f^{-1}$ extends to an orthogonal automorphism $\Phi$ on $\mathfrak{g}$ that commutes with the Cartan involution $\sigma$. In this case $\Phi$ can be chosen such that $F=\left.\Phi\right|_{\mathfrak{p}}$.

Proof. " $\Rightarrow$ ": Let $F: \mathfrak{p} \rightarrow \mathfrak{p}$ be an orthogonal linear map extending $f$; this is unique since, by fullness the $K$-orbit $M$ does not lie in a proper linear subspace of $\mathfrak{p}$. We claim that $F$ is $K$-equivariant, that is:

$$
F(k x)=\phi(k) F(x)
$$

for any $k \in K$ and for any $x \in \mathfrak{p}$. Clearly, if $x \in M$ we have $F(k x)=f(k x)=f k f^{-1} f x=$ $\phi(k) F x$. Thus $F=\phi(k) F k^{-1}$ on $M$, and hence $\phi(k) F k^{-1}$ is another orthogonal linear map on $\mathfrak{p}$ which extends $f$. By uniqueness we have $F=\phi(k) F k^{-1}$ and hence $F k=\phi(k) F$ on all of $\mathfrak{p}$.

Now we define a linear map $\Phi$ on $\mathfrak{g}$ putting $\Phi=F$ on $\mathfrak{p}$ and $\Phi=\phi_{*}$ on $\mathfrak{k}$. We claim that $\Phi$ is an orthogonal Lie algebra automorphism of $\mathfrak{g}$. First we show that $\Phi$ is orthogonal with respect to the bi-invariant metric on $\mathfrak{g}$ which restricts to the given metric on $\mathfrak{p}$. By irreducibility of $\mathfrak{p}$, there is just one $\operatorname{Ad}_{K}$-invariant metric on $\mathfrak{p}$ up to a factor, hence we may assume that the metric on $\mathfrak{g}$ is the negative Killing form,

$$
\langle A, B\rangle=-\operatorname{trace}_{\mathfrak{g}}\left(\operatorname{ad}_{A} \circ \operatorname{ad}_{B}\right)
$$

for all $A, B \in \mathfrak{g}$. For $A, B \in \mathfrak{p}$, the scalar product is preserved by $\Phi$ since $\left.\Phi\right|_{\mathfrak{p}}=F$ is orthogonal. Moreover, $\mathfrak{p} \perp \mathfrak{k}$ since $\operatorname{ad}_{A}, A \in \mathfrak{k}$ preserve the decomposition $\mathfrak{g}=\mathfrak{k} \oplus \mathfrak{p}$ while $\operatorname{ad}_{x}, x \in \mathfrak{p}$ exchanges the two factors. Now let $A, B \in \mathfrak{k}$. Then $\operatorname{ad}_{A} \circ \operatorname{ad}_{B}$ preserves $\mathfrak{k}$ and $\mathfrak{p}$, and

$$
\operatorname{trace}_{\mathfrak{g}}\left(\operatorname{ad}_{A} \circ \operatorname{ad}_{B}\right)=\operatorname{trace}_{\mathfrak{k}}\left(\operatorname{ad}_{A} \circ \operatorname{ad}_{B}\right)+\operatorname{trace}_{\mathfrak{p}}\left(\operatorname{ad}_{A} \circ \operatorname{ad}_{B}\right) .
$$

The $\mathfrak{k}$-part is the Killing form of $\mathfrak{k}$ which is invariant under every automorphism of $\mathfrak{k}$. In contrast, the $\mathfrak{p}$-part needs not to be invariant, but our automorphism $\left.\Phi\right|_{\mathfrak{k}}=\phi_{*}$ has a special form: It is conjugation with an orthogonal map $F \in O(\mathfrak{p})$ which normalizes $K \subset O(\mathfrak{p})$. Since the trace is invariant under conjugation, $\phi_{*}$ preserves the $\mathfrak{p}$-part of $(4)$. Hence $\phi_{*}$ is orthogonal on $\mathfrak{k}$ and $\Phi$ orthogonal on $\mathfrak{g}$.

Now we show that $\Phi$ is an automorphism of $\mathfrak{g}$. From the equivariance (Equation (3)) we obtain $F\left(\operatorname{ad}_{B} x\right)=\operatorname{ad}_{\phi_{*}(B)} F x$ for all $B \in \mathfrak{k}$ and $x \in \mathfrak{p}$ and hence

$$
\Phi[B, x]=[\Phi(B), \Phi(x)] .
$$


Moreover, $[x, y] \in \mathfrak{k}$ for all $x, y \in \mathfrak{p}$ and hence $\Phi[x, y]=\phi_{*}[x, y]$ while for all $A \in \mathfrak{k}$ we have, using the orthogonality of $\Phi$ and (5) with $\Phi(B)=A$,

$$
\begin{aligned}
\langle A,[\Phi x, \Phi y]\rangle & =\langle[A, \Phi x], \Phi y\rangle=\left\langle\Phi\left[\Phi^{-1} A, x\right], \Phi y\right\rangle=\left\langle\left[\Phi^{-1} A, x\right], y\right\rangle=\left\langle\Phi^{-1} A,[x, y]\right\rangle \\
& =\langle A, \Phi[x, y]\rangle .
\end{aligned}
$$

Thus $\Phi[x, y]=[\Phi x, \Phi y]$ which shows that $\Phi$ is a Lie algebra automorphism.

" $\Leftarrow$ ": Let $\phi$ be the automorphism of $K$ with $\phi(k)=f k f^{-1}$ as in (2). Suppose that $\phi_{*}: \mathfrak{k} \rightarrow \mathfrak{k}$ extends to an orthogonal automorphism $\Phi$ of $\mathfrak{g}$ which commutes with $\sigma$. Our base point $\xi$ lies in the fixed space $F_{\xi}$ of the group $L=K_{\xi}$,

$$
F_{\xi}=\left\{v \in \mathfrak{p}: k v=v \forall k \in K_{\xi}\right\}=\bigcap_{k \in K_{\xi}} F_{k}
$$

where $F_{k}=\operatorname{Fix}(k)=\{v \in \mathfrak{p}: k v=v\}$ is the fixed space of $k \in K_{\xi}$ in $\mathfrak{p}$. We claim

$$
\Phi\left(F_{\xi}\right)=F_{\xi} .
$$

We have to show $\operatorname{Ad}_{k}(\Phi v)=\Phi v$ for any $k \in K_{\xi}$ and $v \in F_{\xi}$. Since $K$ is connected, we may write $k=\exp A$ for some $A \in \mathfrak{k}$, but not necessarily $A \in \mathfrak{k}_{\xi}$. Then

$$
\Phi^{-1} \circ \operatorname{Ad}_{k} \circ \Phi=\operatorname{Ad}_{\exp \phi_{*}^{-1} A}=\operatorname{Ad}_{\phi^{-1}(k)} .
$$

Thus $\Phi^{-1} \operatorname{Ad}_{k} \Phi v=\operatorname{Ad}_{\phi^{-1}(k)} v=v$ for any $v \in F_{\xi}$ since $k \in K_{\xi}$ and $K_{\xi}$ is preserved by $\phi^{-1}$. This proves claim (6).

Every $k \in K_{\xi}$ commutes with the extrinsic symmetry $\rho_{\xi}$ whose eigenspaces are the tangent and normal spaces $T_{\xi}$ and $N_{\xi}$ of $M$ at $\xi$. Thus the fixed space $F_{\xi}$ of $K_{\xi}$ is also invariant under $\rho_{\xi}$ and therefore it decomposes as $F_{\xi}=F_{\xi}^{T} \oplus F_{\xi}^{N}$ where $F_{\xi}^{T}=F_{\xi} \cap T_{\xi}$ and $F_{\xi}^{N}=F_{\xi} \cap N_{\xi}$. For each $\eta \in F_{\xi}^{N}$ we have $[\eta, \xi]=0$ since

$$
\langle\mathfrak{k},[\xi, \eta]\rangle=\langle[\mathfrak{k}, \xi], \eta\rangle=\left\langle T_{\xi} M, \eta\right\rangle=0 .
$$

Thus for each $\eta \in F_{\xi}^{N}$, there is a maximal abelian subspace $\mathfrak{a} \subset \mathfrak{p}$ containing both $\eta$ and $\xi$. Since $T_{\xi}=[\mathfrak{k}, \xi]$ and $N_{\xi}=[\mathfrak{k}, \xi]^{\perp}=\{x \in \mathfrak{p}:\langle\mathfrak{k},[\xi, x]\rangle=0\}=\{x \in \mathfrak{p}:[\xi, x]=0\}$, we have $\mathfrak{a} \subset N_{\xi}$. We claim that $\operatorname{dim}\left(F_{\xi}^{N} \cap \mathfrak{a}\right)=1$ and hence $\eta \in \mathbb{R} \xi$. In fact, as $\xi$ satisfies (1), it is a vertex of a spherical Weyl chamber in $\mathfrak{a}$, and hence $K \xi$ is a "most singular" orbit (its isotropy group $L=K_{\xi}$ is maximal). Or in other terms: by (1), $\xi$ is a dual root for some simple root system $\alpha_{1}, \ldots, \alpha_{r}$ for $\mathfrak{a}$, say $\xi=\alpha_{j}^{*}$. Let

$$
\mathfrak{k}_{\alpha_{i}}=\left\{a \in \mathfrak{k} \mid \exists b \in \mathfrak{p} \forall x \in \mathfrak{a}:[x, a]=\alpha_{i}(x) b,[x, b]=-\alpha_{i}(x) a\right\}
$$

be the root space in $\mathfrak{k}$ which corresponds to the root $\alpha_{i}$. It follows from $\alpha_{i}(\xi)=0$ that $\mathfrak{k}_{\alpha_{i}} \subset K_{\xi}$ for all $i \neq j$. Thus $\left[\mathfrak{k}_{\alpha_{i}}, \eta\right]=0$ for $\eta \in F_{\xi} \cap \mathfrak{a}$ and therefore $\alpha_{i}(\eta)=0$. Hence $\eta$ as well as $\xi$ belong to the one-dimensional subspace $\bigcap_{i \neq j} \operatorname{ker} \alpha_{i} \subset \mathfrak{a}$ which means $\eta \in \mathbb{R} \xi$. Since $\eta \in F_{\xi}^{N}$ was arbitrary, we conclude that $F_{\xi}^{N}$ is one-dimensional, $F_{\xi}^{N}=\mathbb{R} \xi$.

What about the tangent part $F_{\xi}^{T}$ ? Let us consider $F_{\xi} \cap M$. Two points of $M$, say $k \xi$ and $\xi$, have the same fixed space, $F_{\xi}=F_{k \xi}$, if and only if $k \in N:=N_{K}\left(K_{\xi}\right)=$ the normalizer of $K_{\xi}$ in $K$, and hence $F_{\xi} \cap M=N \xi$. Since $K_{\xi}$ is a normal subgroup of $N$, its Lie algebra $\mathfrak{k}_{\xi}$ is an ideal of $\mathfrak{n}=\operatorname{Lie}(N)$, and also its orthogonal complement (with respect 
to any Ad-invariant metric on $\mathfrak{k})$ is an ideal. Thus $\mathfrak{n}$ splits into ideals, $\mathfrak{n}=\mathfrak{k}_{\xi} \oplus \mathfrak{n}^{\prime}$. The full orthogonal complement $\mathfrak{k}_{\xi}^{\perp} \subset \mathfrak{k}$ is considered as the tangent space of $M=K \xi=K / K_{\xi}$ at $\xi$, and $\mathfrak{n}^{\prime}$ is the subspace corresponding to $F_{\xi}^{T}$. In particular, $F_{\xi}^{T}=0$ if $\mathfrak{n}^{\prime}=0$. Therefore let us assume that $\mathfrak{n}^{\prime} \neq 0$. Since $\mathfrak{n}^{\prime} \subset \mathfrak{k}_{\xi}^{\perp}$ is fixed under $\operatorname{Ad}_{\left(K_{\xi}\right)^{o}}$, the local holonomy group of $M$, any non-zero element of $\mathfrak{n}^{\prime}$ determines locally a parallel vector field on $M$. Hence locally $M$ splits off a one-dimensional factor which gives a global splitting for its universal covering: $\tilde{M}=\mathbb{R} \times \tilde{M}^{\prime}$. Since $K$ is the transvection group of $M$, some covering $\tilde{K}$ of $K$ is the transvection group of $\tilde{M}$, and this splits off a real factor, $\tilde{K}=\mathbb{R} \times \tilde{K}^{\prime}$. Thus $\mathfrak{k}=\mathbb{R} \oplus \mathfrak{k}^{\prime}$. This happens if and only if $\mathfrak{p}$ is the Lie triple of an irreducible hermitian symmetric space and $\mathfrak{n}^{\prime}=\mathbb{R}$ and $F_{\xi}^{T}$ is one-dimensional, see [11, Chapter VIII, section 6].

Thus $F_{\xi}$ is either one-dimensional and normal to $M$, or it is two dimensional and $F_{\xi} \cap M$ is a circle. In both cases, $F_{\xi}=\bigcup_{\eta \in M \cap F_{\xi}} F_{\eta}^{N}$. Therefore $\Phi(\xi) \in F_{\eta}^{N}$ for some $\eta \in M \cap F_{\xi}$, and hence $\Phi(\xi)= \pm \eta= \pm \operatorname{Ad}_{n} \xi$ for some $n \in N^{\prime}$. If the sign is " + ", we put $\tilde{\Phi}=\operatorname{Ad}_{n^{-1}} \circ \Phi$, and if the sign is "-", we let $\tilde{\Phi}=\sigma \circ \operatorname{Ad}_{n^{-1}} \circ \Phi$ where $\sigma$ is the Cartan involution on $\mathfrak{g}$. In both cases, $\tilde{\Phi}=\Phi=\phi_{*}$ on $\mathfrak{k}$ since $n^{-1}$ lies in the center of $K$. Thus $\tilde{\Phi} \xi=\xi$. Now we put $F=\left.\tilde{\Phi}\right|_{\mathfrak{p}}$. For any $x \in \mathfrak{p}$ and $k \in K$,

$$
\tilde{\Phi}\left(\operatorname{Ad}_{k} x\right)=\operatorname{Ad}_{\phi(k)} \tilde{\Phi} x
$$

as in (7). Applying (8) to $x=\xi$ we obtain

$$
\tilde{\Phi}(k \xi)=\tilde{\Phi}\left(\operatorname{Ad}_{k} \xi\right)=\operatorname{Ad}_{\phi(k)} \tilde{\Phi} \xi=\operatorname{Ad}_{\phi(k)} \xi=\phi(k) \xi
$$

for all $k \in K$. But also $f(k \xi)=f k f^{-1} f \xi=\phi(k) \xi$, hence $\left.\tilde{\Phi}\right|_{M}=f$, as desired.

We now apply Proposition 5.3 to the geodesic symmetry $f=s_{\xi}$ of $M$ at the point $\xi$. By definition of an extrinsic symmetric space $s_{\xi}$ extends to the ambient vector space $\mathfrak{p}$. In this case the corresponding automorphism

$$
\tau: K \rightarrow K, \quad k \mapsto s_{\xi} k s_{\xi}
$$

of $K$, is called Cartan involution for the symmetric space $M$.

Corollary 5.4. Let $s_{\xi}$ be the geodesic symmetry of $M$ at $\xi$ and $\tau \in \operatorname{Aut}(K)$ the conjugation with the symmetry. Then $\tau_{*}$ extends to an orthogonal automorphism of $\mathfrak{g}$.

Corollary 5.5. Let $M=K \xi \subset V$ be a non-hermitean indecomposable extrinsic symmetric space and $L=K_{\xi}$ its isotropy group. Assume that one of the following conditions holds:

(i) K has no outer automorphism.

(ii) L has no outer automorphism and the isotropy representation of $M=K / L$ is irreducible.

Then every isometry $f$ of $M$ extends to an orthogonal map of $V$.

Proof. Let $f$ be an isometry of $M$. As before, we may assume that $f$ fixes $\xi$. Therefore the automorphism $\phi$ of $K$ given by the conjugation with $f$ preserves $L$, that is $\phi(L)=L$.

(i): If $K$ has no outer automorphism, then $\phi_{*}$ is inner automorphism of $\mathfrak{k}$ which clearly extends to an inner automorphism of $\mathfrak{g}$, thus we are done by Proposition 5.3. 
(ii): If $L$ has no outer automorphism, then $\left.\phi\right|_{L}$ is inner. Thus there is an inner automorphism $\tilde{\phi}$ of $K$ by conjugation with an element of $L$ such that $\tilde{\phi}=\phi$ on $L$. Hence $\psi=\tilde{\phi} \phi^{-1}$ is an automorphism of $K$ fixing every point in $L$. Let $\mathfrak{k}=\mathfrak{m} \oplus \mathfrak{l}$ be the Cartan decomposition corresponding to $M=K / L$. Then $\psi_{*}$ commutes with the isotropy representation of $L$ on $\mathfrak{m}$. Since this representation is irreducible and not complex, Schur's lemma implies $\psi_{*}= \pm I$ on $\mathfrak{m}$. Thus either $\phi$ or $\tau \circ \phi$ are inner, where $\tau$ is the Cartan involution on $K$ corresponding to $M$. In both cases, $\phi_{*}$ extends to $\mathfrak{g}$ (where we use Corollary 5.4 in the second case), and we are done by Proposition 5.3.

Outer automorphisms of a simply connected compact semi-simple Lie group correspond to automorphisms of its Dynkin diagram; more precisely, every non-trivial diagram automorphism corresponds to a class of outer automorphisms modulo composition with inner automorphisms and vice versa (see e.g. [18, Proposition 3.4, p. 128]). For each outer automorphism $\tau$ of the universal covering $\tilde{K}$ of $K$ we will ask the following questions:

(1) Does $\tau$ descend to an automorphism of $K$ ?

(2) If yes, does $\tau$ keep $L$ invariant?

(3) If yes, does $\tau_{*}$ extend to a Lie algebra automorphism of $\mathfrak{g}$ ?

The answer to (1) is the same for every automorphism in the same class. An automorphism for which the first three questions are answered affirmatively is called admissible. We must show that (3) is answered affirmatively for any admissible automorphism $\tau$ of $\tilde{K}$.

5.3.1. Cases No. 5 - 8. By $\tilde{K}$ and $\tilde{L}$ we denote the universal covers of $K$ and $L$. If they do not admit outer automorphisms, the same holds for $K$ and $L$. As the Dynkin diagrams of type $C_{n}$ and $F_{4}$ do not allow for diagram automorphisms we get:

Case No. $5: \quad \tilde{K}=S p_{n}$ has no outer automorphism.

Case No. 6: $\quad \tilde{K}=S p_{4}$ has no outer automorphism.

Case No. $7: \quad \tilde{K}=F_{4}$ has no outer automorphism.

Case No. 8: $\quad \tilde{L}=S p_{4}$ has no outer automorphism and the isotropy representation of $K / L$ is irreducible.

We are done by Corollary 5.5.

5.3.2. Case No. 9: $M=G_{p}\left(\mathbb{R}^{n}\right)$. In this case $M=S O_{n} / S\left(O_{p} \times O_{q}\right)$ with $p+q=n$; this is the Grassmannian $G_{p}\left(\mathbb{R}^{n}\right)$ of $p$-planes in $\mathbb{R}^{n}$, embedded in the space of symmetric matrices. If $n=2 m+1$ is odd, the Dynkin diagram of $\tilde{K}=\operatorname{Spin}_{n}$ is of type $B_{m}$ and has no non-trivial automorphisms. Thus all automorphisms of $K$ are inner for $n$ odd.

If $n=2 m$ is even, then $K=S O_{2 m} / \pm=\operatorname{Spin}_{2 m} / Z$ where $Z$ denotes the center of $\operatorname{Spin}_{2 m}$. Thus all automorphisms of $\operatorname{Spin}_{2 m}$ descend to $K$. The Dynkin diagram is of type $D_{m}$, and there is always a non-trivial diagram automorphism, the reflection $\rho$ exchanging the two ends of the bifurcation of $D_{m}$. 


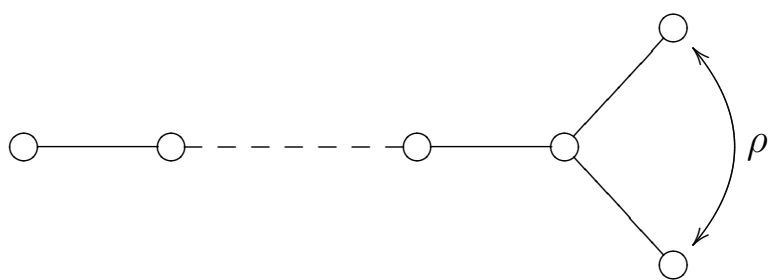

Conjugation with a hyperplane reflection on $\mathbb{R}^{2 m}$ is an outer automorphism (see Lemma 5.6 below) of order 2 on $S O_{2 m}$ and hence on $S O_{2 m} / \pm$. This conjugation clearly extends to the group $G=S U_{2 m} / \pm$ that has Lie algebra $\mathfrak{s u}_{2 m}$. For $m \neq 4$ we are done since there are no other diagram automorphisms of $D_{m}$.

It remains to consider the case $m=4, n=8$. The automorphism group of the Dynkin diagram $D_{4}$ is the dihedral group $S_{3}$ of the equilateral triangle, which is generated by the reflection $\rho$ and a rotation of the three branches of $D_{4}$. A rotation corresponds to the triality automorphism $\theta$ of $\operatorname{Spin}_{8}$ and $S O_{8} / \pm$. In Lemma 5.7, see the next section 5.4, we show that no automorphism in the class of $\theta$ preserves $L=S\left(O_{p} \times O_{8-p}\right) / \pm$. Thus the only admissible outer automorphism are in the class of $\rho$. Hence the case $m=4$ is not special. This finishes case 9 .

Lemma 5.6. Let $A \in O_{n}$ with $\operatorname{det} A=-1$ and let $\alpha(X)=A X A^{-1}$ for any $X \in S O_{n}$. Then $\alpha$ is an inner (outer) automorphism of $S O_{n}$ if $n$ is odd (even).

Proof. Suppose that $\alpha$ is inner. Then there is some $B \in S O_{n}$ with $A X A^{-1}=B X B^{-1}$ for all $X \in S O_{n}$, and $C:=B^{-1} A$ commutes with every $X \in S O_{n}$. Since $S O_{n} \subset \mathbb{C}^{n \times n}$ is an irreducible complex representation, we have $C= \pm I$ by Schur's lemma (any complex eigenspace of $C$ is $S O_{n}$-invariant, hence the real matrix $C$ has only one eigenvalue). Thus $B= \pm A$, but $\operatorname{det} B=1$ and $\operatorname{det} A=-1$ which holds if and only if $B=-A$ and $n$ is odd.

\subsection{The triality automorphism.}

This section is self-contained. For references and further details see e.g. $[1,10,15]$.

Recall that the Clifford algebra $C l_{8}$ is the algebra of all real $16 \times 16$ matrices

$$
C l_{8}=\mathbb{R}^{16 \times 16}=\left\{\left(\begin{array}{cc}
A & B \\
C & D
\end{array}\right) \mid A, B, C, D \in \mathbb{R}^{8 \times 8}\right\} .
$$

We have $C l_{8}=C l_{8}^{+} \oplus C l_{8}^{-}$where the "even" and the "odd" parts $C l_{8}^{ \pm}$consist of the block diagonal and block anti-diagonal matrices $\left({ }^{A}{ }_{D}\right)$ and $\left({ }_{C}{ }^{B}\right)$, respectively. The vector space $\mathbb{R}^{8}=\mathbb{O}$ is embedded into $C l_{8}^{-}$as

$$
\widehat{\mathbb{O}}=\left\{\widehat{x}:=\left(\begin{array}{ll} 
& -L_{\bar{x}} \\
L_{x} &
\end{array}\right): x \in \mathbb{O}\right\}
$$

where $\mathbb{O}=\mathbb{R}^{8}$ is the normed division algebra of the octonions and $L_{x} \in \mathbb{R}^{8 \times 8}$ the left multiplication with any $x \in \mathbb{O}$. Note that

$$
\widehat{x} \widehat{y}=\left(\begin{array}{ll}
-L_{\bar{x}} L_{y} & \\
& -L_{x} L_{\bar{y}}
\end{array}\right)
$$


which implies the Clifford relation $\widehat{x} \widehat{y}+\widehat{y} \widehat{x}=-2\langle x, y\rangle I_{16}$, using the octonionic relations

$$
L_{\bar{x}} L_{y}+L_{\bar{y}} L_{x}=2\langle x, y\rangle I_{8}
$$

which arise from polarizing $L_{\bar{x}} L_{x}=\langle x, x\rangle I_{8}$.

The group $\mathrm{Pin}_{8}$ is the subgroup of the multiplicative group of $C l_{8}$ generated by the subset $\widehat{\mathbb{S}^{7}}=\left\{\widehat{v}: v \in \mathbb{S}^{7}\right\} \subset C l_{8}$. Conjugation with $\widehat{v}$ preserves $\widehat{\mathbb{O}}$; in fact, up to sign it is the reflection $S_{v}$ at the hyperplane $v^{\perp}$ since

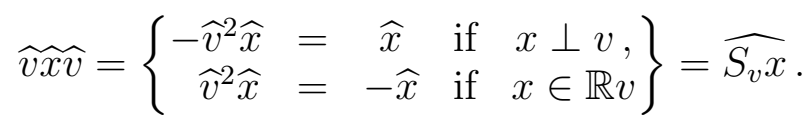

The group $\operatorname{Spin}_{8}$ is the subgroup generated by the set of matrices

$$
\left\{\widehat{v} \widehat{w}=\left({ }_{B}^{A}\right): A=-L_{\bar{v}} L_{w}, B=-L_{v} L_{\bar{w}}, v, w \in \mathbb{S}^{7}\right\} \subset C l_{8}^{+} .
$$

Conjugation with $\widehat{v} \widehat{w}=\left({ }_{B}^{A}\right)$ preserves $\widehat{\mathbb{O}}$ and corresponds to a rotation $C \in S O_{8}$,

$$
\left(\begin{array}{ll}
A_{B} &
\end{array}\right) \widehat{x}\left(\begin{array}{ll}
A^{t} & \\
B^{t}
\end{array}\right)=\widehat{C x}
$$

or equivalently

$$
B L_{x}=L_{C x} A .
$$

In particular, for the generators (9) of $\operatorname{Spin}_{8}$ we have

$$
A=-L_{\bar{v}} L_{w}, B=-L_{v} L_{\bar{w}} \Rightarrow C=S_{v} S_{w} .
$$

Applying both sides of (10) to any $y \in \mathbb{O}$, we obtain for all $x, y \in \mathbb{O}$

$$
B(x y)=(C x)(A y) .
$$

This property (12) of the octonions is known as triality principle. Let

$$
H=\left\{(A, B, C) \in S O_{8} \times S O_{8} \times S O_{8}: B(x y) \stackrel{(12)}{=}(C x)(A y) \forall x, y \in \mathbb{O}\right\} .
$$

This is a subgroup of $S O_{8} \times S O_{8} \times S O_{8}$ : If $(A, B, C),\left(A^{\prime}, B^{\prime}, C^{\prime}\right) \in H$, then

$$
B^{\prime} B(x y) \stackrel{(12)}{=} B^{\prime}((C x)(A y)) \stackrel{(12)^{\prime}}{=}\left(C^{\prime} C x\right)\left(A^{\prime} A y\right),
$$

hence $\left(A A^{\prime}, B B^{\prime}, C C^{\prime}\right) \in H$. Further,

$$
B^{-1}(x y)=B^{-1}\left(C C^{-1} x\right)\left(A A^{-1} y\right) \stackrel{(12)}{=}\left(C^{-1} x\right)\left(A^{-1} y\right),
$$

thus $\left(A^{-1}, B^{-1}, C^{-1}\right) \in H$.

By (12), the homomorphism $\pi_{12}:(A, B, C) \mapsto(A, B)$ takes values in $\operatorname{Spin}_{8} \subset S O_{8} \times S O_{8}$. In fact, $\pi_{12}: H \rightarrow \operatorname{Spin}_{8}$ is an isomorphism: It is injective since $(A, B)$ determines $C$ by (12). Further, applying (12) in the cases $x=1 \in \mathbb{O}$ or $y=1$ and putting $a=A(1)$ and $c=C(1)$ we see $B=L_{c} A$ and $B=R_{a} C$ where $L$ and $R$ denote left and right multiplication in $\mathbb{O}$. Consequently, $L_{c} A=R_{a} C$ and hence $C=R_{a}^{-1} L_{c} A$. Consider the homomorphism $\pi_{3}: H \rightarrow S O_{8},(A, B, C) \mapsto C$. We claim that ker $\pi_{3}=\{(I, I, I),(-I,-I, I)\}$. In fact, when $C=I$, then $c=1$ and $A=B=R_{a}$, thus $(x y) \stackrel{(12)}{=} x(y a)$ for all $x, y \in \mathbb{O}$. This associativity holds only for $a \in \mathbb{R}$, and since $|a|=1$ we obtain $a= \pm 1$. Consequently, 
$\pi_{12}(H) \subset \operatorname{Spin}_{8}$ is a 2-fold cover of $S_{8}$ which proves $\pi_{12}(H)=\operatorname{Spin}_{8}$. Therefore we will no longer distinguish between $H$ and $\operatorname{Spin}_{8}$. The map

$$
\pi_{3}: H=\operatorname{Spin}_{8} \rightarrow S O_{8}:(A, B, C) \mapsto C
$$

is the so called vector representation of Spin $_{8}$.

The triality automorphism $\theta$ on $\operatorname{Spin}_{8}$ is essentially the cyclic permutation of triples $(A, B, C) \in \operatorname{Spin}_{8}$. More precisely: assuming $|x|=|y|=1$ and putting $z=x y$, we have

$$
(C x)(A y)=B z \Longleftrightarrow(\overline{A y})(\overline{C x})=\overline{B z} \Longleftrightarrow \overline{C x}=(A y)(\overline{B z}) .
$$

Now $\overline{C x}=\tilde{C} \bar{x}$ with $\tilde{C}=\kappa C \kappa$, where $\kappa x=\bar{x}$ is the octonionic conjugation, $\kappa=\left({ }^{-1}{ }_{I_{7}}\right)$. Thus (12) holds if and only if $\tilde{C} \bar{x}=(A y)(\tilde{B} \bar{z})$, and since $y \bar{z}=\bar{x}$ when $z, x$ have unit length, we have $(\tilde{B}, \tilde{C}, A) \in \operatorname{Spin}_{8}$. The map

$$
\theta:(A, B, C) \mapsto(\tilde{B}, \tilde{C}, A)
$$

is the triality automorphism of $\operatorname{Spin}_{8}$. It has order 3 since its iterations are

$$
(A, B, C) \mapsto(\tilde{B}, \tilde{C}, A) \mapsto(C, \tilde{A}, \tilde{B}) \mapsto(A, B, C) .
$$

This automorphism of $\operatorname{Spin}_{8}$ descends to an automorphism of Spin 8 /center $=S O_{8} / \pm$, called $\theta$ again, and using (13) and (11) we obtain

$$
\theta: \pm C \mapsto \pm A, \quad \pm S_{v} S_{w} \mapsto \pm L_{\bar{v}} L_{w} .
$$

But $\theta$ does not descend to $\mathrm{SO}_{8}$ since changing $w$ to $-w$ does not change $C$ while it does change the sign of $A$. Thus $\theta$ cannot be an inner automorphism. Since it has order 3 , it must lie in the class of a diagram automorphisms of order 3 for the Dynkin diagram $D_{4}$.

Lemma 5.7. The subgroup $L=S\left(O_{p} \times O_{8-p}\right) / \pm$ of $K=S O_{8} / \pm$ is not invariant under the automorphism $\gamma \theta$ for any inner automorphism $\gamma$ of $K$.

Proof. We proceed by contradiction. Suppose that $L$ is invariant under $\gamma \theta$ for some inner automorphism $\gamma(X)=g^{-1} X g, X \in S O_{8}$. We have $L=\left\{ \pm k \in K: k\left(\mathbb{R}^{p}\right)=\mathbb{R}^{p}\right\}$. From $\gamma \theta L=L$ we obtain $\theta L=g L g^{-1}=\left\{ \pm k \in K: k\left(g \mathbb{R}^{p}\right)=g \mathbb{R}^{p}\right\}$. Thus all elements of $\theta(L)$ leave the subspace $g \mathbb{R}^{p} \subset \mathbb{R}^{8}$ invariant. But $L$ contains the elements $S_{e_{1}} S_{e_{i}}$ for $i=2, \ldots, 8$ which are mapped by $\theta$ onto $L_{e_{1}} L_{e_{i}}=L_{e_{i}}$ using (14) (recall that $e_{1}=1 \in \mathbb{O}$ ). Thus the subspace $V=g \mathbb{R}^{p} \subset \mathbb{O}$ must be invariant under all $L_{e_{i}}$, hence $V$ is invariant under left multiplications with all octonions. But any non-zero subspace $V \subset \mathbb{O}$ with this property is the full division algebra $\mathbb{O}$ : Left multiplication of any non-zero $v \in V$ with $v^{-1}$ gives 1 , hence $1 \in V$, and then $x=L_{x} 1 \in V$ for any $x \in \mathbb{O}$.

Remark 5.8. Note that in the case $p=4$, the identity component of $L$ is preserved by $\theta$ : In fact, this is $L_{+}:=\left(S O_{4} \times S O_{4}\right) / \pm$, which is generated by $\pm S_{v} S_{w}$ with either $v, w \in \mathbb{R}^{4}=\mathbb{H}$ (the standard quaternion subalgebra in $\mathbb{O}$ ) or $v, w \in \mathbb{H}^{\perp}$. In both cases, $\pm L_{\bar{v}} L_{w}$ also preserves $\mathbb{H}$ and thus belongs to $L_{+}$. Thus $\theta\left(L_{+}\right)=L_{+}$which shows that $\theta$ induces an isometry on the Grassmannian of oriented 4-planes $G_{4}^{+}\left(\mathbb{R}^{8}\right)$. But only the Grassmannian $G_{4}\left(\mathbb{R}^{8}\right)$ of non-oriented 4 -planes is extrinsic symmetric, not $G_{4}^{+}\left(\mathbb{R}^{8}\right)$. 


\subsection{Cases with local $\mathbb{S}^{1}$-factor.}

It remains to consider cases No. 10, 11 and 12 with $K=K^{\prime} \cdot \mathbb{S}^{1}=\left(K^{\prime} \times \mathbb{S}^{1}\right) / \Gamma$, where the Lie algebra $\mathfrak{k}^{\prime}$ of $K^{\prime}$ is simple and $L \subset K^{\prime}$. Both $K^{\prime}$ and $\mathbb{S}^{1}$ can be considered as transformation groups on $\mathfrak{p}$, where $\mathbb{S}^{1}=\{\lambda I: \lambda \in \mathbb{C},|\lambda|=1\}$ and $\Gamma=K^{\prime} \cap \mathbb{S}^{1}$. Then $\mathbb{S}^{1} \subset K$ is the center of $K$. Thus any automorphism $\phi$ of $K$ preserves the subgroups $\mathbb{S}^{1}$ and $K^{\prime}$; observe that $\mathfrak{k}^{\prime}=[\mathfrak{k}, \mathfrak{k}]$ is invariant under $\phi_{*}$. Let $\alpha$ and $\beta$ be the automorphisms on $K^{\prime}$ and $\mathbb{S}^{1}$ induced by $\phi$. There are only two possibilities for $\beta$, the identity $\beta(\lambda)=\lambda$ or the complex conjugation $\beta(\lambda)=\bar{\lambda}$. The possibilities for $\alpha$ are obtained from the Dynkin diagrams of $\mathfrak{k}^{\prime}$ and $\mathfrak{l}$. But we also need to have $\alpha=\beta$ on $\Gamma=K^{\prime} \cap \mathbb{S}^{1}$. In all but one case, $\phi$ is inner or the Cartan involution $\tau$ for $M=K / L$, and then $\phi_{*}$ extends to an automorphism of the larger Lie algebra $\mathfrak{g}$, using Corollary 5.4. This finishes the proof using Proposition 5.3 .

5.5.1. Case No. 10: $K=\left(E_{6} \times \mathbb{S}^{1}\right) / \mathbb{Z}_{3}, L=S p_{4}$. Since the Dynkin diagram $C_{4}$ of $S p_{4}$ has no non-trivial diagram automorphism, any automorphism $\alpha$ of $K^{\prime}=E_{6}$ preserving $L$ is inner on $L$. After composing $\alpha$ with an inner automorphism if necessary, we may assume $\left.\alpha\right|_{L}=\mathrm{id}$, and hence $\alpha_{*}$ commutes with $\operatorname{Ad}_{L}$. Since the isotropy representation of $K^{\prime} / L$ is irreducible, we have by Schur's Lemma (see the proof of Corollary 5.5) $\alpha_{*}=$ id or $\alpha_{*}$ is the Cartan involution $\tau^{\prime}$ for $K^{\prime} / L$. If $\beta(\lambda)=\bar{\lambda}$, the two non-trivial center elements are interchanged, thus $\alpha=\tau^{\prime}$, and $(\alpha, \beta)$ is the Cartan involution $\tau$ for $M=K / L$. This extends to $\mathfrak{g}$ by Corollary 5.4. If $\beta(\lambda)=\lambda$, we need to have $\alpha$ inner, and this automorphism extends, too.

5.5.2. Case No. 11: $K=U_{2 n} / \pm, L=S p_{n} / \pm, n \geq 3$. Again the Dynkin diagram $C_{n}$ does not allow for any non-trivial diagram automorphism. Thus we may assume $\left.\alpha\right|_{L}=\mathrm{id}$, and as before we conclude $\alpha \in\left\{\mathrm{id}, \tau^{\prime}\right\}$ where $\tau^{\prime}$ is the Cartan involution for $K^{\prime} / L=S U_{2 n} / S p_{n}$. We have $U_{2 n} / \pm=\left(S U_{2 n} \times \mathbb{S}^{1}\right) / \widehat{\Gamma}$ where $\widehat{\Gamma}=\left\{(\zeta I, \pm \zeta): \zeta^{2 n}=1\right\}$. Let us assume $\alpha=$ id and $\beta(\lambda)=\bar{\lambda}$. Then we need to have $\zeta= \pm \bar{\zeta}$ for all $\zeta$ with $\zeta^{2 n}=1$, but this is true only for $n=2$ which was excluded.* In all other cases this is impossible. The only remaining possibilities for $\phi$ are either the identity or the Cartan involution $\tau$ for $K / L$. Thus $\phi_{*}$ extends to $\mathfrak{g}$, cf. Corollary 5.4.

5.5.3. Case No. 12: $K=U_{n} / \pm, L=O_{n} / \pm, n \geq 3$. As in the previous case, the only automorphisms of $S U_{n} \times \mathbb{S}^{1}$ which descend to $U_{n} / \pm$ are inner automorphisms and the Cartan involution $\tau$ for $K / L$, unless $n \neq 4$. But on $U_{4} / \pm$ there is yet another automorphims: $\phi( \pm A)= \pm A / \sqrt{\operatorname{det} A}$. In fact, $\phi=$ id on $S U_{4}$ and $\operatorname{det}(\phi(A))=\operatorname{det}(A) / \sqrt{\operatorname{det}(A)^{4}}=$ $1 / \operatorname{det} A$, hence $\phi(\lambda)=\bar{\lambda}$ for $\lambda \in \mathbb{S}^{1}$. But $\phi$ is not admissible: it fixes the identity component $L_{+}=S O_{4} / \pm$ of $L=O_{4} / \pm$, but the other component $L_{-}=\left\{ \pm A: A \in O_{4}\right.$, $\left.\operatorname{det} A=-1\right\}$ is not mapped to $L$ as $\sqrt{\operatorname{det} A}$ is no longer real. Likewise, $\gamma \phi$ cannot preserve $L$ for any inner

\footnotetext{
${ }^{*}$ In the case $n=2$ we have $K^{\prime} / L=\left(S U_{4} / \pm\right) /\left(S p_{2} / \pm\right)=S O_{6} / S O_{5}=\mathbb{S}^{5}$, and $K / L=\left(\mathbb{S}^{5} \times \mathbb{S}^{1}\right) / \pm$; this belongs to case 4 , see Section 5.2.3.
} 
automorphism $\gamma(x)=g x g^{-1}$ on $U_{4}$ : Otherwise, $\gamma$ would preserve $L_{+}$, but any conjugation in $U_{4}$ preserving $L_{+}$would also preserve $L$ while $\phi$ does not preserve $L$, a contradiction.

This finishes the proof of theorems 1.1 and 2.1.

\section{REFERENCES}

[1] J. F. Adams, Lectures on exceptional Lie Groups, University of Chicago Press, Chicago 1996

[2] J. Berndt, S. Console and C. Olmos, Submanifolds and holonomy, Chapman Hall/CRC Res. Notes in Math. 434, Chapman \& Hall/CRC, Boca Raton 2003

[3] J.-H. Eschenburg and E. Heintze, Extrinsic symmetric spaces and orbits of s-representations, Manuscr. Math. 88 (1995), 517-524

[4] J.-H. Eschenburg, P. Quast and M. S. Tanaka, Isometries of hermitian symmetric spaces, J. Lie Theory 23 (2013), 113-118

[5] J.-H. Eschenburg, P. Quast and M. S. Tanaka, Maximal tori of extrinsic symmetric spaces and meridians, Osaka J. Math. 52 (2015), 299-305

[6] D. Ferus, Immersionen mit paralleler zweiter Fundamentalform: Beispiele und Nicht-Beispiele, Manuscr. Math. 12 (1974), 153-162

[7] D. Ferus, Immersions with parallel second fundamental form, Math. Z. 140 (1974), 87-93

[8] D. Ferus, Produkt-Zerlegung von Immersionen mit paralleler zweiter Fundamentalform, Math. Ann. 211 (1974), 1-5

[9] D. Ferus, Symmetric submanifolds of euclidean space, Math. Ann. 247 (1980), 81-93

[10] F. R. Harvey, Spinors and Calibrations, Academic Press, New York 1990

[11] S. Helgason, Differential geometry, Lie groups and symmetric spaces, Pure Appl. Math. 80, Academic Press, New York 1978

[12] U. Hirzebruch, Über eine Realisierung der hermiteschen, symmetrischen Räume, Math. Z. 115 (1970), 371-382

[13] S. Kobayashi, Isometric imbeddings of compact symmetric spaces, Tohoku Math. J. 20 (1968), 21-25

[14] S. Kobayashi and T. Nagano, On filtered Lie algebras and geometric structures I, J. Math. Mech. 13 (1964), 875-907

[15] H. B. Lawson and M.-L. Michelsohn, Spin Geometry, Princeton University Press, Princeton 1990

[16] D. S. P. Leung, On the classification of reflective submanifolds of Riemannian symmetric spaces, Indiana Univ. Math. J. 24 (1974), 327-339

[17] A. Lichnérowicz, Géométrie des groupes de transformations, Dunod, Paris 1958

[18] O. Loos, Symmetric spaces II: compact spaces and classification, W. A. Benjamin, New York 1969

[19] A.-L. Mare and P. Quast, Bott periodicity for inclusions of symmetric spaces, Doc. Math., J. DMV 17 (2012) 911-952

[20] A.-L. Mare and P. Quast, On some spaces of minimal geodesics in Riemannian symmetric spaces, Q. J. Math. 63 (2012), 681-694

[21] T. Nagano, Transformation groups on compact symmetric spaces, Trans. Amer. Math. Soc. 118 (1965), 428-453

[22] P. Quast, A geometric proof of a result of Takeuchi, Tohoku Math. J. (2) 66 (2014), 427-434

[23] M. Takeuchi, Cell decompositions and Morse equalities on certain symmetric spaces, J. Fac. Sci., Univ. Tokyo, Sect. I 12 (1965), 81-191

[24] M. Takeuchi and S. Kobayashi, Minimal imbeddings of $R$-spaces, J. Differ. Geom. 2 (1968), 203-215

[25] M. Takeuchi, Stability of certain minimal submanifolds of compact hermitian symmetric spaces, Tohoku Math. J. 36 (1984), 293-314

[26] McK. Wang and W. Ziller, Symmetric spaces and strongly isotropy irreducible spaces, Math. Ann. 296 (1993), 285-326 
(Eschenburg \& Quast) Institut für Mathematik, Universität Augsburg, 86135 Augsburg, GERMANY

E-mail address, Eschenburg: eschenburg@math.uni-augsburg.de

E-mail address, Quast: peter.quast@math.uni-augsburg.de

(Tanaka) Faculty of Science and Technology, Tokyo University of Science, Noda, Chiba, 278-8510, JAPAN

E-mail address, Tanaka: tanaka_makiko@ma.noda.tus.ac.jp 